\title{
Längsschnittstudie zum Verlauf motorischer Fähigkeiten von Grundschulkindern in Abhängigkeit auffälliger motorischer Leistungen der Fein- und Grobmotorik
}

\author{
Andreas Speer ${ }^{1}$, Petra Wagner ${ }^{1}$, Heike Streicher ${ }^{1}$, Alexandra Ziegeldorf ${ }^{1}$, Ines Benkert ${ }^{2}$ \\ und Hagen Wulff ${ }^{1}$
}

'Institut für Gesundheitssport und Public Health, Universität Leipzig

${ }^{2}$ Gesundheitsamt der Stadt Leipzig

\begin{abstract}
Zusammenfassung: Theoretischer Hintergrund: Die motorische Leistungsfähigkeit (MLF) spielt eine zentrale Rolle in der Kindesentwicklung. Über den Verlauf der MLF über die Grundschulzeit in Abhängigkeit auffälliger motorischer Leistungen im Vorschulalter liegen nur wenige Befunde vor. Fragestellung: Liegen unterschiedliche Entwicklungsverläufe der MLF bei Kindern mit motorisch auffälligen Leistungen in der Fein- und Grobmotorik vor? Methode: Innerhalb einer Längsschnittstudie wurden die motorischen Dimensionen Kraft, Ausdauer, Schnelligkeit, Koordination und Beweglichkeit von Grundschulkindern ( $N=424)$ jährlich untersucht und mittels Varianzanalyse mit Messwiederholung geprüft. Ergebnisse: Kinder, die vor Schuleintritt grob- oder feinmotorische Auffälligkeiten (9-15\%) aufwiesen, blieben in ihrer motorischen Entwicklung deutlich hinter motorisch unauffälligen Kindern zurück. Diskussion und Schlussfolgerung: In der Folge können sich erhebliche Einschränkungen für die Altagsmotorik und das Erlernen komplexer Bewegungen ergeben. Um gleiche motorische Startbedingungen für die betroffenen Kinder herzustellen, stellt die Erweiterung der bewegungsbezogenen Förderung der MLF vor Schulbeginn einen notwendigen Ansatz dar.
\end{abstract}

Schlüsselwörter: Längsschnitt, motorische Leistungsfähigkeit, auffällige motorische Leistung, Kinder

A Longitudinal Study of Motor Ability Development of Primary-School-Aged Children with Developmental Fine and Gross Motor Disorders

Abstract: Theoretical Background: Motor performance is an important matter in the health-related development of children, particularly for perception and for establishing a personal and material environment using physical activity. Developmental coordination disorders in preschoolaged children may relate to lower levels of fine and gross motor development. Short-term longitudinal studies revealed that preschoolers with motor deficits fall behind in their overall motor performance during the 1st and 2nd grades of primary school. Moreover, the years at primary school are a meaningful stage in life for children because of its rapid progression in motor-learning capability. Objective: Regarding children in primary school, little is known about the effects of developmental coordination disorders on the grade-related progression of basic motor abilities (i.e., flexibility, strength, endurance, speed of movement, coordination). This study analyzes the motor performance development of children over the period of primary school. Method: Using a longitudinal study design (KOMPASS-2 Study), we examined motor ability development in a sample of $N=424$ primary school-aged children ( $n=218$ girls, 51.4\%). To assess levels of motor abilities, we used the German Motor Test (DMT 6-18). Based on standard screening for school entry, children were separated into two groups based on the status of their fine and gross motor development. Changes in motor ability levels were analyzed via robust repeated measures analyses of variance (rmANOVA) regarding developmental group effects, school timeframe effects, and interaction effects. Results: $9 \%(n=39)$ of the children were classified with a gross motor disorder, and $15 \%(n=62)$ of the children were classified with a fine motor disorder. The statistical analyses with rmANOVA demonstrated that children with developmental coordination disorders regarding gross or fine motor developmental status showed lower motor-ability levels on all test tasks compared to children without disorders. Particularly gross-motor disordered children achieved significantly lower motor-ability levels regarding coordination under time constraint (jumping sideways) and coordination during dynamic precision tasks (backward balancing). However, motor-ability levels increased significantly over time for both developmental status groups. Discussion and conclusion: Children with developmental coordination disorders may experience substantial restrictions to meeting daily physical activities and motor learning of complex movements. To create equal motor developmental conditions for children just starting school, it is necessary to promote physical activity in general. Interventions should regard a set of coordination tasks that require children's attention and speed during movement. Measuring the motor abilities of primary-school-aged children once a year should be an integral part of communal health monitoring.

Keywords: longitudinal study, motor performance, developmental coordination disorder, primary school children 
Die Förderung der motorischen Leistungsfähigkeit durch tägliche Bewegung stellt einen wirksamen Schutzfaktor vor physischen und psychischen Erkrankungen dar und ist deshalb ein zentraler Baustein der Gesundheitsförderung in der Kindheit und Jugend (Gabrys, 2017). Gerade im Grundschulalter lässt sich aufgrund der Reifungs- und Wachstumsprozesse der Kinder ein schneller Zuwachs motorischer Leistungen beobachten (Albrecht, HanssenDoose, Bös et al., 2016). Zur Charakterisierung und Interpretation motorischer Entwicklungsverläufe im Grundschulalter haben sich verschiedene theoretische Rahmenkonzepte etabliert (Scheuer, Herrmann \& Bund, 2019). Auf der Basis des in der Sportwissenschaft am weitesten verbreiteten Konzepts, dem Strukturmodell sportmotorischer Fähigkeiten (Utesch, Zinner \& Büsch, 2018), wird die motorische Leistungsfähigkeit in energetisch determinierte und informatorisch determinierte Voraussetzungen der Bewegungshandlungen gegliedert. Auf der untergeordneten Ebene erfolgt die Differenzierung in die Basisfähigkeiten Ausdauer, Kraft, Schnelligkeit und Koordination, wobei die Beweglichkeit als passives System der Energieübertragung eine eigenständige Basisfähigkeit darstellt (Bös, 2016). Mit Hilfe unterschiedlicher motorischer Einzeltests zu diesen Basisfähigkeiten lässt sich eine objektive Erfassung und Einschätzung der Motorik durch einen Vergleich mit altersund geschlechtsspezifischen Referenzwerten vornehmen (Utesch et al., 2018).

Unterdurchschnittlich motorische Leistungen werden im Kindesalter als eine der bedeutsamsten Entwicklungsabweichungen gesehen (Kastner \& Petermann, 2010a) und können zudem mit einer umschriebenen Entwicklungsstörung motorischer Funktionen (UEMF) (F82 nach ICD-10) assoziiert sein. Diese geht mit erheblichen Einschränkungen im alltäglichen Funktionsniveau einher und lässt sich nicht auf sensorische, kognitive oder neurologische Defizite zurückführen (Dilling, Mombour, Schmidt et al., 2016). Mit einer Prävalenz von etwa 5-6\% zählen sie zu einer der häufigsten Entwicklungsauffälligkeiten im Vor- und Grundschulter (Blank \& Vinçon, 2020; Werpup-Stüwe \& Petermann, 2015). Während fundierte Kenntnisse zur Entwicklung der motorischen Leistungsfähigkeit im mittleren Kindesalter und damit in der Grundschulzeit vorliegen (Woll, Worth, Bös et al., 2019) sind längsschnittliche Betrachtungen in Abhängigkeit auffälliger motorischer Leistungen nur vereinzelt vorhanden (Krombholz, 2015), beziehen sich auf ausgewählte Fähigkeitskomplexe wie zum Beispiel koordinative Leistungen (Losse, Henderson, Elliman et al., 1991) oder sind auf andere Konstrukte wie die Vorhersage motorischer Kompetenzen ausgerichtet (Baumann, Tresilian, Bartmann et al., 2020). Rivilis et al. (2011) stellte in einem systematischen Review fest, dass niedrige motorische
Leistungen unter anderem negativ mit dem Niveau der kardiorespiratorischen Fitness, der Muskelkraft sowie der Kraftausdauer assoziiert sind. Sie verweisen jedoch darauf, dass die Beobachtungszeiträume eher gering und Vergleiche aufgrund unterschiedlicher Ansätze auffälliger motorische Leistungen nur bedingt möglich sind. Aktuelle Studien unterstützen die Befunde dahingehend, dass motorische Auffälligkeiten von fünf- und sechsjährigen Kindern über den Zeitabschnitt der Vorschule und Einschulung zeitlich stabil bleiben (Michel, Molitor, Fahmer et al., 2019). Wie sich die Kinder in den folgenden Schuljahren motorisch entwickeln, bleibt offen. Zudem fokussieren bisherige empirische Analysen vorrangig das motorische Gesamtniveau der Kinder anhand eines aggregierten Motorikindizes oder Motorikquotienten (Krombholz, 2015; Ružbarská, 2016). Das Vorschul- und Grundschulalter ist im besonderen Maße durch eine deutliche $\mathrm{Zu}-$ nahme des Bewegungskönnens gekennzeichnet (Winter \& Hartmann, 2015). Im Übergang zum mittleren Kindesalter findet zudem eine weitere qualitative Ausdifferenzierung der motorischen Fähigkeitskomplexe statt, die sich für die Bereiche der Kraft, Ausdauer und Schnelligkeit auch faktoranalytisch einordnen lässt (Utesch, Strauß, Tietjens et al., 2015). Vor diesem Hintergrund hat die vorliegende Studie das Ziel, die motorische Entwicklung von Vorschulkindern längsschnittlich über den Zeitraum der Grundschule zu analysieren. Um der altersspezifischen Ausdifferenzierung der motorischen Fähigkeitskomplexe im mittleren Kindesalter gerecht zu werden, wird ausgehend von Ergebnissen der Schuleingangsuntersuchung eine differenzierte Betrachtung der motorischen Fähigkeiten in Abhängigkeit des fein- und grobmotorischen Entwicklungsstands der Vorschulkinder vorgenommen. Mit einer differenzierten Analyse der motorischen Leistung ließen sich auch zukünftige Programme zur motorischen Bewegungsförderung spezifischer an die Bedarfe der Kinder anpassen. Aufgrund der bisher dargestellten Forschungsergebnisse wird erwartet, dass sich motorische Auffälligkeiten vor Schulbeginn auch in unterschiedlichen Entwicklungsverläufen der motorischen Basisfähigkeiten Kraft, Ausdauer, Schnelligkeit, Koordination und Beweglichkeit über die Grundschulzeit widerspiegeln.

\section{Methoden}

\section{Studiendesign}

Von November 2013 bis Mai 2014 wurde im Rahmen der kommunalen Schuleingangsuntersuchungen einmalig der motorische Entwicklungsstand der Kinder durch das Ge- 
Tabelle 1. Stichprobenumfang und -häufigkeiten differenziert nach Alter und Geschlecht

\begin{tabular}{|c|c|c|c|c|c|c|c|c|c|c|c|}
\hline \multirow[b]{3}{*}{ Erhebun } & & \multirow{2}{*}{\multicolumn{2}{|c|}{$\begin{array}{c}\text { Gesamt } \\
\text { Alter (Jahre) }\end{array}$}} & \multicolumn{4}{|c|}{ Mädchen } & \multicolumn{4}{|c|}{ Jungen } \\
\hline & & & & \multicolumn{2}{|c|}{ Fälle } & \multicolumn{2}{|c|}{ Alter (Jahre) } & \multicolumn{2}{|c|}{ Fälle } & \multicolumn{2}{|c|}{ Alter (Jahre) } \\
\hline & & $M$ & $S D$ & $n$ & $\%$ & $M$ & $S D$ & $n$ & $\%$ & $M$ & $S D$ \\
\hline SEU & $(N=665)$ & 5.96 & 0.35 & 332 & 49.9 & 5.93 & 0.35 & 333 & 50.1 & 5.99 & 0.35 \\
\hline \multirow{4}{*}{$\begin{array}{l}\text { Längsschnitt } \\
\text { KOMPASS-2-Studie }\end{array}$} & $\begin{array}{l}\text { 1. Klasse } \\
(N=1140)\end{array}$ & 6.99 & 0.39 & 579 & 50.8 & 6.98 & 0.39 & 561 & 49.2 & 7.02 & 0.39 \\
\hline & $\begin{array}{l}\text { 2. Klasse } \\
(N=1135)\end{array}$ & 7.97 & 0.39 & 575 & 50.7 & 7.96 & 0.39 & 560 & 49.3 & 7.98 & 0.39 \\
\hline & $\begin{array}{l}\text { 3. Klasse } \\
(N=1019)\end{array}$ & 9.00 & 0.41 & 516 & 50.6 & 9.00 & 0.43 & 503 & 49.4 & 9.01 & 0.39 \\
\hline & $\begin{array}{l}\text { 4. Klasse } \\
(N=1008)\end{array}$ & 9.98 & 0.41 & 504 & 50.0 & 9.95 & 0.41 & 504 & 50.0 & 9.99 & 0.41 \\
\hline
\end{tabular}

Anmerkungen: $M$ = Mittelwert, $S D$ = Standardabweichung, SEU = Schuleingangsuntersuchung, $N=$ Gesamtstichprobenumfang, $n=$ Teilstichprobenumfang.

sundheitsamt der Stadt Leipzig erhoben. Die längsschnittliche Erfassung der motorischen Leistungsfähigkeit fand in den Schuljahren 2014/15 bis 2017/18 statt (KOMPASS-2-Studie). 30 von 65 Leipziger Grundschulen nahmen daran teil (Streicher, Wulff, Hartmann et al., 2017). Eine Nichtteilnahme wurde durch die Schulen unter anderem mit einer eingeschränkten Nutzung der Sportanlagen begründet. Die Studie umfasst insgesamt fünf Erhebungszeitpunkte, von der Schuleingangsuntersuchung im Vorschulalter und den jährlichen MotorikTests von der 1. Klasse bis zur 4. Klasse.

\section{Stichprobe}

Zu Studienbeginn wurden Einverständniserklärungen zur freiwilligen Teilnahme an die Eltern versendet, innerhalb derer über die Studieninhalte und Zielstellung informiert wurde. Es konnten nur Kinder teilnehmen, deren $\mathrm{Zu}$ stimmung der Eltern vorlag. Zum Zeitpunkt der 1. Klasse konnten insgesamt $N=1140$ Grundschulkinder $(n=579$ Mädchen, 51\%) rekrutiert werden (Tab. 1).

Die Drop-out-Rate betrug bis zum Zeitpunkt der 4 . Klasse $12 \%$. Gründe für den Ausstieg waren ein Wohnortwechsel oder der Schulwechsel an eine nichtteilnehmende Grundschule. Über das Gesundheitsamt Leipzig konnte uns aus Kapazitätsgründen ein Datensatz mit $N=$ 665 Kindern ( $n=332$ Mädchen; 49,9\%) zur Verfügung gestellt werden, der anschließend über interne Identifikationsnummern mit dem Datensatz der 1. Klasse synchronisiert wurde. Über diesen Datenabgleich wurde eine abhängige Stichprobe mit $N=424$ Kindern generiert. Diese setzte sich aus $n=218$ Mädchen (51,4\%) (Response: $65,7 \% ; 5,91 \pm 0,36$ Jahre; $1,18 \pm 0,05 \mathrm{~m} ; 21,20 \pm 2,87 \mathrm{~kg})$ und $n=206$ Jungen (48,6\%) (Response: 61,9\%; 5,97 \pm 0,35 Jahre; 1,19 $\pm 0,06 \mathrm{~m} ; 22,16 \pm 3,41 \mathrm{~kg})$ zusammen.

\section{Datenerhebung}

\section{Motorischer Entwicklungstand im Vorschulalter}

Als Untersuchungsverfahren wurde das Screening des Entwicklungsstandes (S-ENS) eingesetzt (Döpfner, Dietmair, Mersmann et al., 2005). In diesem werden die Einzeltests Visuomotorik und Körperkoordination verwendet, die in ihrer Aufgabenstruktur den übergeordneten Kategorien der Fein- und Grobmotorik zugeordnet werden können. Der Visuomotoriktest umfasste zwei Testaufgaben zum Nachzeichnen und Ergänzen geometrischer Figuren. Zur Einschätzung der Grobmotorik mussten die Kinder die Testaufgabe beidbeiniges seitliches Hin- und Herspringen absolvieren. Die Messergebnisse wurden in Anlehnung an eine Zweiteilung nach Molitor et al. (2015) sowie die Befundungskala des S-ENS-Testmanuals (Döpfner et al., 2005) in auffällig (einschließlich grenzwertig) (Feinmotorik: definiert durch einen Punktwert $\leq 16$, Grobmotorik: definiert durch eine Sprungzahl $\leq 8$ ) und unauffällig dichotomisiert. Die vier resultierenden motorischen Entwicklungsgruppen (MEG) werden als feinmotorisch unauffällig, feinmotorisch auffällig, grobmotorisch unauffällig und grobmotorisch auffällig zusammengefasst. In Anlehnung an die Testinstruktionen des S-ENS (Döpfner, Dietmair, Mersmann et al., 2004) wird bei den MEG nicht zwischen Mädchen und Jungen unterschieden. Döpfner et al. (2005) verweisen auf eine zufriedenstellende bis sehr gute konvergente Validität der Merkmalsbereiche des SENS hin. Die Werte der internen Konsistenz (Cronbachs a) liegen bei den Untertests des S-ENS zwischen 0,50 und 0,76 .

\section{Motorische Leistungsfähigkeit über die Grundschulzeit}

Die motorischen Fähigkeiten wurden mit dem Deutschen Motoriktest (DMT 6-18) ermittelt (Bös, 2016). Dieser 
umfasst acht Testaufgaben: $20 \mathrm{~m}$-Sprint (Aktionsschnelligkeit), Standweitsprung (Schnellkraft bei Sprüngen), Balancieren rückwärts (Koordination bei Präzisionsaufgaben), seitliches Hin- und Herspringen (Koordination unter Zeitdruck bei Sprüngen), Sechs-Minuten-Lauf (aerobe Ausdauer beim Laufen), Rumpfbeuge (Rumpfbeweglichkeit), Sit-ups (Kraftausdauer der Rumpfmuskulatur) und Liegestütz (Kraftausdauer der oberen Extremitäten). Die Testdurchführung fand einmal jährlich zu Beginn des Schuljahres innerhalb des Sportunterrichts statt. Die Hauptgütekriterien wurden erfolgreich überprüft (Bös, 2016). Fehlende Werte wurden mithilfe multipler Imputationsverfahren nach den Empfehlungen von Jekauc, Völkle, Lämmle et al. (2012) ersetzt.

\section{Statistische Auswertung}

Die Auswertung erfolgt mit R (Version 3.6.3) und SPSS (Version 25). $\mathrm{Zu}$ den auffälligen fein- und grobmotorischen Leistungen werden die Prävalenzen berichtet. Die Leistungen des Visuomotorik- und Körperkoordinationstests der Schuleingangsuntersuchung werden mittels TTest für unabhängige Stichproben auf Unterschiedlichkeit geprüft $(p<.05)$. Zur Beschreibung der Motorik werden die Mittelwerte (M) und die Standardabweichung (SD) berichtet. Die Unterschiedsprüfung erfolgt aufgrund von Homogenitätsverletzungen über eine robuste Varianzanalyse mit Messwiederholung (ANOVAR) (Mair \& Wilcox, 2020) jährlich von der 1. bis zur 4. Klasse (1. Haupteffekt: MEG, 2. Haupteffekt: Zeit; Interaktionseffekt: MEG ${ }^{*}$ Zeit $)(p<.05)$. Die Effektgröße partielles $\eta^{2}$ wird wie folgt interpretiert: $.010<\eta^{2} \leq .060$ klein; $.060<\eta^{2}$ $\leq .140$ mittel; $\eta^{2}>.140$ groß (Cohen, 1988). Beim Vorliegen signifikanter Effekte werden post-hoc-Tests (Yuen's trimmed mean t-Test) mit Bonferroni-Holm-Adjustierung ergänzt.

\section{Ergebnisse}

\section{Prävalenz der auffälligen fein- und grobmotorischen Leistungen}

Bei der Auswertung der Daten zur Grobmotorik konnten lediglich 421 Fälle einbezogen werden, da drei Kinder den Test verweigerten. Im Bereich der Feinmotorik zeigen $15 \%(n=62)$, bei der Grobmotorik $9 \%(n=39)$ der Kinder ein auffälliges Testergebnis. Die Prüfung der Mittelwerte ergab, dass sich die beiden MEG sowohl bei der Grobmotorik (Anzahl der korrekten Sprünge) $(t(419)=11.503$, $p<.001)$ als auch bei der Feinmotorik (Gesamtpunktzahl der fehlerfreien Strichführung) $(t(70.097)=18.850, p<$ .001) deutlich voneinander unterscheiden. Dies spricht für die diskriminante Validität der Gruppenbildung und die interne Validität der Gruppenvergleiche in Bezug auf die motorischen Entwicklungsverläufe.

\section{Unterschiede im Verlauf der motorischen Fähigkeiten in Abhängigkeit auffälliger grobmotorischer Leistungen}

Die deskriptiven Kennzahlen der MEG sind in Tabelle 2 dargestellt.

Bei allen Testaufgaben liegen signifikante Veränderungen über die Zeit mit Steigerungen der Leistungen über die vier Klassenstufen vor $(p<.05)$. Die Ergebnisse der ANOVAR sind in Tabelle 3 zusammengefasst und entsprechen nahezu linearen Leistungsanstiegen.

Beim Rumpfbeugetest geht die Entwicklung sowohl mit Leistungsverbesserungen als auch -verschlechterungen einher. Die Post-hoc-Tests ergeben bis zur 3. Klasse einen signifikanten Leistungsanstieg (1. Klasse vs. 3. Klasse: $p<$ $\left..001, p_{\text {adjustiert }}<.008\right)$. Danach liegen deutlich niedrigere Leistungen vor (3. Klasse vs. 4 . Klasse: $p<.001$, $p_{\text {adjustiert }}<$ .010). Zwischen der 1. Klasse und 4. Klasse lassen sich wiederum keine Unterschiede nachweisen $\left(p=.367\right.$, $p_{\text {adjus }}$ tiert $<.05)$. Bis auf die Testleistung Rumpfbeuge entsprechen alle Leistungssteigerungen über die Zeit mittleren bis großen Effekten $\left(.24<\eta^{2}<76\right)$ mit der größten Entwicklung beim seitlichen Hin- und Herspringen (Zeit: $F=$ 436.2, $\left.p<.001, \eta^{2}=.76\right)$. Im Vergleich der Gruppeneffekte haben bis auf die Testaufgabe Rumpfbeuge motorisch unauffällige Kinder signifikant höhere Leistungen als motorisch auffällige Kinder. Der größte Gruppenunterschied liegt bei der Testaufgabe Balancieren rückwärts vor (MEG: $F=25.5, p<.001, \eta^{2}=.15$ ). Ergänzende Informationen zu den grafischen Entwicklungsverläufen sind in der Abbildung 1a-h im Elektronischen Supplement 1 dieses Beitrags hinterlegt.

\section{Unterschiede im Verlauf der motorischen Fähigkeiten in Abhängigkeit der auffälligen feinmotorischen Leistungen}

Alle Kinder verbessern sich über die vier Jahre bis auf die Testaufgabe Rumpfbeuge. Die Zeiteffekte sind mittel bis groß $\left(.22<\eta^{2}<76\right)$. Die größte Leistungsentwicklung findet beim seitlichen Hin- und Herspringen statt (Zeit: $F$ $=519.2, p<.001, \eta^{2}=0.76$ ) (siehe Tab. 2 und Tab. 3). Motorisch unauffällige Kinder erreichen bei sechs von acht Testaufgaben deutlich höhere Leistungen $(p<.05)$ als motorisch auffällige Kinder. Am bedeutsamsten sind 
Tabelle 2. Deskriptive Kennzahlen zu den motorischen Fähigkeiten für die motorischen Entwicklungsgruppen der Grob- und Feinmotorik

\begin{tabular}{|c|c|c|c|c|c|c|c|c|c|}
\hline \multirow[t]{2}{*}{ Test } & \multirow[t]{2}{*}{ Erhebung } & \multicolumn{2}{|c|}{$\begin{array}{l}\text { grob-a } \\
(n=39)\end{array}$} & \multicolumn{2}{|c|}{$\begin{array}{l}\text { grob-u } \\
(n=382)\end{array}$} & \multicolumn{2}{|c|}{$\begin{array}{c}\text { fein-a } \\
(n=62)\end{array}$} & \multicolumn{2}{|c|}{$\begin{array}{c}\text { fein-u } \\
(n=362)\end{array}$} \\
\hline & & $M$ & $S D$ & $M$ & $S D$ & $M$ & $S D$ & $M$ & $S D$ \\
\hline \multirow[t]{4}{*}{20 m (s) } & 1. Kl. & 5.06 & 0.44 & 4.84 & 0.36 & 4.94 & 0.44 & 4.84 & 0.35 \\
\hline & 2. Kl. & 4.90 & 0.45 & 4.66 & 0.33 & 4.81 & 0.43 & 4.66 & 0.33 \\
\hline & 3. Kl. & 4.65 & 0.36 & 4.44 & 0.34 & 4.54 & 0.34 & 4.44 & 0.34 \\
\hline & 4. Kl. & 4.54 & 0.37 & 4.36 & 0.32 & 4.55 & 0.34 & 4.35 & 0.32 \\
\hline \multirow[t]{4}{*}{ SW (m) } & 1. Kl. & 1.07 & 0.17 & 1.15 & 0.17 & 1.07 & 0.15 & 1.16 & 0.17 \\
\hline & 2. Kl. & 1.16 & 0.17 & 1.26 & 0.17 & 1.22 & 0.21 & 1.26 & 0.16 \\
\hline & 3. Kl. & 1.23 & 0.19 & 1.31 & 0.18 & 1.25 & 0.18 & 1.31 & 0.18 \\
\hline & 4. Kl. & 1.29 & 0.19 & 1.40 & 0.19 & 1.30 & 0.20 & 1.41 & 0.19 \\
\hline \multirow[t]{4}{*}{$\overline{\mathrm{SHH}}($ Anzahl) } & 1. Kl. & 14.8 & 4.3 & 18.2 & 4.4 & 16.0 & 4.1 & 18.3 & 4.5 \\
\hline & 2. $\mathrm{Kl}$. & 19.7 & 6.1 & 24.0 & 5.1 & 21.8 & 5.0 & 23.9 & 5.3 \\
\hline & 3. Kl. & 24.9 & 6.5 & 29.5 & 5.5 & 26.5 & 6.0 & 29.5 & 5.6 \\
\hline & 4. Kl. & 30.6 & 6.3 & 34.8 & 5.3 & 32.1 & 5.6 & 34.8 & 5.4 \\
\hline \multirow[t]{4}{*}{ Bal rw (Schritte) } & 1. Kl. & 20.4 & 11.2 & 25.8 & 9.1 & 22.2 & 9.9 & 25.8 & 9.2 \\
\hline & 2. Kl. & 26.5 & 9.2 & 33.2 & 9.2 & 29.2 & 10.6 & 33.1 & 9.0 \\
\hline & 3. Kl. & 28.6 & 8.6 & 34.7 & 8.8 & 32.2 & 9.5 & 34.4 & 8.8 \\
\hline & 4. Kl. & 30.6 & 10.3 & 37.7 & 8.1 & 34.0 & 9.3 & 37.6 & 8.3 \\
\hline \multirow[t]{4}{*}{ 6-Min (m) } & 1. Kl. & 871.0 & 127.3 & 919.0 & 117.4 & 878.7 & 107.5 & 920.2 & 120.0 \\
\hline & 2. Kl. & 926.2 & 120.3 & 976.1 & 120.9 & 923.6 & 137.0 & 979.3 & 116.6 \\
\hline & 3. Kl. & 988.2 & 197.1 & 1023.1 & 129.7 & 970.4 & 137.7 & 1028.0 & 135.4 \\
\hline & 4. Kl. & 1009.0 & 156.5 & 1058.1 & 126.2 & 1002.7 & 147.4 & 1062.5 & 124.3 \\
\hline \multirow[t]{4}{*}{$\mathrm{RB}(\mathrm{cm})$} & 1. Kl. & 0.3 & 7.3 & 0.0 & 6.3 & -1.0 & 7.2 & 0.3 & 6.2 \\
\hline & 2. Kl. & -0.2 & 7.8 & 0.8 & 6.8 & -0.3 & 7.9 & 0.9 & 6.7 \\
\hline & 3. Kl. & 1.1 & 8.5 & 1.3 & 7.4 & 0.0 & 8.5 & 1.6 & 7.4 \\
\hline & 4. Kl. & -1.0 & 9.6 & 0.2 & 7.8 & -2.5 & 8.6 & 0.6 & 7.7 \\
\hline \multirow[t]{4}{*}{$\overline{S U}($ Anzahl) } & 1. Kl. & 10.5 & 6.5 & 13.2 & 5.2 & 10.7 & 5.9 & 13.4 & 5.2 \\
\hline & 2. Kl. & 12.3 & 6.7 & 15.7 & 4.8 & 13.6 & 6.4 & 15.7 & 4.8 \\
\hline & 3. Kl. & 15.6 & 6.7 & 18.7 & 4.7 & 16.4 & 5.6 & 18.8 & 4.8 \\
\hline & 4. Kl. & 18.5 & 6.2 & 20.9 & 4.9 & 19.0 & 6.0 & 21.0 & 4.8 \\
\hline \multirow[t]{4}{*}{ LS (Anzahl) } & 1. Kl. & 9.1 & 3.7 & 10.9 & 3.3 & 9.5 & 3.9 & 11.0 & 3.2 \\
\hline & 2. Kl. & 10.1 & 4.0 & 12.9 & 3.3 & 11.6 & 3.9 & 12.8 & 3.4 \\
\hline & 3. Kl. & 12.9 & 4.5 & 15.4 & 3.6 & 14.5 & 3.9 & 15.3 & 3.8 \\
\hline & 4. Kl. & 14.4 & 4.3 & 16.3 & 3.6 & 15.3 & 3.5 & 16.3 & 3.8 \\
\hline
\end{tabular}

Anmerkungen: $M=$ Mittelwert, $S D=$ Standardabweichung, $n=$ Teilstichprobenumfang, $K$ l. = Klasse, fein- $u=$ feinmotorisch unauffällig, fein-a = feinmotorisch auffällig, grob-u = grobmotorisch unauffällig, grob-a = grobmotorisch auffällig, $20 \mathrm{~m}=20 \mathrm{~m}$-Sprint, SW = Standweitsprung, Bal $\mathrm{rw}=$ Balancieren rückwärts, SHH = Seitliches Hin- und Herspringen, 6-Min = Sechs-Minuten-Lauf, RB = Rumpfbeuge, SU = Sit-ups, LS = Liegestütz.

die Gruppendifferenzen beim Sprint und beim seitlichen Hin- und Herspringen mit mittleren Effekten $\left(.06<\eta^{2}<\right.$ .07). Bei der Testaufgabe Rumpfbeuge konnte eine signifikante Interaktion zwischen der zeitlichen Veränderung und der Gruppenzugehörigkeit festgestellt werden (MEG Zeit: $\left.F=3.4, p=0.032, \eta^{2}=0.02\right)$. Im Ergebnis der Post-hoc-Tests zeigen motorisch auffällige Kinder von der 2. Klasse zur 4. Klasse einen signifikanten Leistungsrückgang $\left(p<.001, p_{\text {adjustiert }}<.008\right)$; während bei den motorisch unauffälligen Kindern keine Leistungsverringerung nachweisbar ist $\left(p<.468, p_{\text {adjustiert }}<.05\right)$.

\section{Diskussion}

Das Ziel der Studie war es, die Entwicklung der motorischen Fähigkeiten von Kindern über die Grundschulzeit in Abhängigkeit ihres motorischen Entwicklungsstands im Vorschulalter zu analysieren. In einer Längsschnittuntersuchung mit jährlichen Erhebungen in jeder Klassenstufe wurden zwei motorische Entwicklungsgruppen von Vorschulkindern mit oder ohne Auffälligkeiten in der Fein- und Grobmotorik auf unterschiedliche Entwicklungsverläufe der motorischen Fähigkeiten geprüft. 
Tabelle 3. Ergebnisse der ANOVAR zur Entwicklung der motorischen Fähigkeiten über die Grundschulzeit

\begin{tabular}{|c|c|c|c|c|c|c|c|c|c|c|c|}
\hline \multirow[b]{2}{*}{ Test } & \multirow[b]{2}{*}{ Effekte } & \multicolumn{5}{|c|}{ Grobmotorik } & \multicolumn{5}{|c|}{ Feinmotorik } \\
\hline & & $F$ & $d f 1$ & $d f 2$ & $p$ & $\eta^{2}$ & $F$ & $d f 1$ & $d f 2$ & $p$ & $\eta^{2}$ \\
\hline \multirow[t]{3}{*}{20 m (s) } & MEG & 14.5 & 1 & 27.5 & 0.00 & 0.09 & 10.5 & 1 & 34.0 & 0.00 & 0.06 \\
\hline & Zeit & 67.9 & 3 & 23.9 & 0.00 & 0.33 & 90.1 & 3 & 26.9 & 0.00 & 0.36 \\
\hline & MEG*Zeit & 0.7 & 3 & 23.9 & 0.56 & 0.01 & 1.1 & 3 & 26.9 & 0.36 & 0.00 \\
\hline \multirow[t]{3}{*}{ SW (m) } & MEG & 13.6 & 1 & 28.7 & 0.00 & 0.09 & 7.1 & 1 & 33.2 & 0.01 & 0.04 \\
\hline & Zeit & 54.0 & 3 & 24.3 & 0.00 & 0.28 & 64.4 & 3 & 26.2 & 0.00 & 0.29 \\
\hline & MEG*Zeit & 1.2 & 3 & 24.3 & 0.35 & 0.02 & 0.4 & 3 & 26.2 & 0.77 & 0.00 \\
\hline \multirow[t]{3}{*}{ SHH (Anzahl) } & MEG & 19.7 & 1 & 26.9 & 0.00 & 0.12 & 11.9 & 1 & 31.4 & 0.00 & 0.07 \\
\hline & Zeit & 436.2 & 3 & 23.5 & 0.00 & 0.76 & 519.2 & 3 & 26.1 & 0.00 & 0.76 \\
\hline & MEG $\star$ Zeit & 1.8 & 3 & 23.5 & 0.19 & 0.01 & 2.1 & 3 & 26.1 & 0.13 & 0.01 \\
\hline \multirow[t]{3}{*}{ Bal rw (Schritte) } & MEG & 25.5 & 1 & 28.3 & 0.00 & 0.15 & 9.1 & 1 & 30.6 & 0.00 & 0.05 \\
\hline & Zeit & 45.3 & 3 & 24.0 & 0.00 & 0.24 & 123.8 & 3 & 28. 9 & 0.00 & 0.44 \\
\hline & MEG*Zeit & 0.2 & 3 & 24.0 & 0.92 & 0.00 & 1.1 & 3 & 28.9 & 0.35 & 0.00 \\
\hline \multirow[t]{3}{*}{$6-\operatorname{Min}(\mathrm{m})$} & MEG & 7.1 & 1 & 29.1 & 0.01 & 0.05 & 4.3 & 1 & 34.4 & 0.05 & 0.03 \\
\hline & Zeit & 65.4 & 3 & 24.2 & 0.00 & 0.32 & 43.7 & 3 & 25.7 & 0.00 & 0.22 \\
\hline & MEG*Zeit & 0.35 & 3 & 24.2 & 0.86 & 0.01 & 0.3 & 3 & 25.7 & 0.85 & 0.00 \\
\hline \multirow[t]{3}{*}{$\mathrm{RB}(\mathrm{cm})$} & MEG & 0.5 & 1 & 26.7 & 0.49 & 0.00 & 1.0 & 1 & 30.2 & 0.34 & 0.01 \\
\hline & Zeit & 4.24 & 3 & 25.1 & 0.02 & 0.03 & 0.8 & 3 & 26.8 & 0.51 & 0.01 \\
\hline & MEG*Zeit & 1.9 & 3 & 25.1 & 0.16 & 0.01 & 3.4 & 3 & 26.8 & 0.03 & 0.02 \\
\hline \multirow[t]{3}{*}{ SU (Anzahl) } & MEG & 8.4 & 1 & 26.1 & 0.01 & 0.06 & 2.7 & 1 & 31.4 & 0.11 & 0.02 \\
\hline & Zeit & 72.5 & 3 & 22.8 & 0.00 & 0.34 & 147.8 & 3 & 26.5 & 0.00 & 0.48 \\
\hline & MEG $\star Z$ eit & 0.5 & 3 & 22.8 & 0.72 & 0.00 & 3.5 & 3 & 26.5 & 0.03 & 0.02 \\
\hline \multirow[t]{3}{*}{ LS (Anzahl) } & MEG & 19.2 & 1 & 26.5 & 0.00 & 0.01 & 4. 5 & 1 & 29.5 & 0.04 & 0.03 \\
\hline & Zeit & 49.4 & 3 & 22.6 & 0.00 & 0.26 & 95.0 & 3 & 26.2 & 0.00 & 0.37 \\
\hline & MEG*Zeit & 0.3 & 3 & 22.6 & 0.82 & 0.00 & 0.2 & 3 & 26.2 & 0.87 & 0.00 \\
\hline
\end{tabular}

Anmerkungen: $\mathrm{MEG}$ = motorische Entwicklungsgruppe, $20 \mathrm{~m}$ = $20 \mathrm{~m}$-Sprint, SW = Standweitsprung, Bal rw = Balancieren rückwärts, SHH = Seitliches Hinund Herspringen, 6-Min = Sechs-Minuten-Lauf, RB = Rumpfbeuge, SU = Sit-ups, LS = Liegestütz.

Die Stichprobe war repräsentativ für die Stadt Leipzig. Trotz der methodischen Einschränkung bei der Synchronisation der Daten für die abhängige Stichprobe konnte eine große Fallzahl von $N=424$ Kindern über jede Klassenstufe der Grundschule untersucht werden. Der Anteil der Mädchen (51,4\%) und Jungen (48,6\%) ist vergleichbar mit aktuellen Längsschnittstudien (Roth, Schmidt, Seidel et al., 2018) und als repräsentativ für diese Altersgruppe einzustufen. Somit ist davon auszugehen, dass die untersuchte Stichprobe eine solide Datengrundlage darstellt.

In Folge von Limitationen bei der Rekrutierung der Stichprobe kann nicht ausgeschlossen werden, dass vermehrt Kinder aus Haushalten mit hoher Bewegungsaffinität der Studienteilnahme zugestimmt haben. Dieser Selektionsbias kann zur Überschätzung der motorischen Entwicklungsverläufe führen. Zudem wurde die Motivation der Kinder bei der Durchführung der motorischen Tests nicht kontrolliert. Hierdurch sind Unterschätzungen im motorischen Entwicklungsverlauf möglich. Eine diffe- renzierte Auswertung der Entwicklungsverläufe nach Geschlecht wurde nicht vorgenommen, was ebenfalls mit einer Verzerrung der Ergebnisse einhergehen kann. So dominieren im mittleren Kindesalter Jungen bei der Kraft, Schnelligkeit und Ausdauer, Mädchen hingegen bei Koordinationsaufgaben und der Beweglichkeit (Roth et al., 2018). Zudem liegen in der Literatur gesicherte Kenntnisse zur Wirkung sozialer Einflussfaktoren vor, die in der Studie keine Berücksichtigung fanden. In diesem Zusammenhang verweisen Krug, Worth, Finger et al. (2019) darauf, dass ein mittlerer und hoher Sozialstatus sowie die Vereinssportaktivität mit einer überdurchschnittlichen motorischen Leistungsfähigkeit korrelieren.

Insgesamt zeigt sich, dass 10-15\% der Kinder der Leipziger Kohorte auffällige motorische Leistungen in der Feinund Grobmotorik aufweisen. Diese Kinder erreichen fast durchgängig schlechtere motorische Leistungen über die Grundschulzeit als motorisch unauffällige Kinder. Somit bleiben auffällige motorische Leistungen im Vorschulalter als Defizite der motorischen Leistungsfähigkeit bis zur 
4. Klasse erhalten. Dies kann zu erheblichen Einschränkungen in der Schule bei alltagsmotorischen Anforderungen wie zum Beispiel dem Tragen des Schulranzens führen. Werden die Rückstände langfristig nicht aufgeholt, besteht die Gefahr, dass motorisch auffällige Kinder durch Überforderung weniger am Sport teilnehmen und dann im Jugend- oder Erwachsenenalter nicht mehr mit Bewegungsangeboten erreicht werden können. Dies hat zur Konsequenz, dass vielfältige Körpererfahrungen ausbleiben und das Erkrankungsrisiko steigt.

Etwa $10 \%$ der Kinder weisen auffällige motorische Leistungen bei der Grobmotorik und $15 \%$ bei der Feinmotorik auf. Das ist vergleichbar mit Beobachtungen von Kastner und Petermann (2010b), nach denen 11-14\% aller Vorschulkinder motorisch-koordinative Störungen aufwiesen. Der Nachweis signifikanter motorischer Leistungsverbesserungen für beide Entwicklungsgruppen über die Grundschulzeit steht im Einklang mit der Literatur. So konnte Krombholz (2015) deutliche Leistungssteigerungen bei Vorschulkindern unabhängig von auffälligen motorischen Leistungen feststellen. Das bedeutet, dass motorische Auffälligkeiten im Vorschulalter vorerst keinen Einfluss auf den zeitlichen Verlauf der motorischen Fähigkeiten über die Grundschulzeit haben. Zudem verläuft die Entwicklung der motorischen Fähigkeiten charakteristisch für das Grundschulalter und stimmt mit Ergebnissen zu Trierer Grundschulkindern überein (Roth et al., 2018).

Dass die Unterschiede der motorischen Entwicklungsverläufe in Abhängigkeit grobmotorischer Entwicklungsstörungen größer ausfallen als in Bezug zur Feinmotorik, wird durch querschnittliche Analysen von Erstklässlern gestützt (Oelze, 2014). Grobmotorisch auffällige Kinder liegen besonders bei Koordinationsaufgaben wie Balancieren rückwärts und seitliches Hin- und Herspringen zurück, die ein hohes Maß an Aufmerksamkeit und Bewegungskontrolle verlangen. Wahrscheinlich fällt es den motorisch auffälligen Kindern bei diesen exekutiven Anforderungen schwerer, Störreize auszublenden oder unerwünschte Bewegungen zu unterdrücken (Molitor et al., 2015). Statistisch sichere Zusammenhänge zwischen der Verarbeitungsgeschwindigkeit und der visuell-räumlichen Verarbeitung (Jaščenoka, Walter, Petermann et al., 2018) unterstreichen diese Annahme.

Da die Bewegungskoordination eine wichtige Voraussetzung für motorische Lernprozesse darstellt (Schnabel, 2015), können motorisch auffällige Kinder erhebliche Nachteile bei der Aneignung alltags- oder sportmotorischer Fertigkeiten wie dem Fahrradfahren oder Schwimmen aufweisen. Das hat zur Konsequenz, dass höhere Gefahren beim Fortbewegen im Wasser oder beim $\mathrm{Zu}$ rücklegen des Schulwegs mit dem Fahrrad bestehen (Speer, Wagner, Streicher et al., 2020).
Motorisch auffällige Kinder, die im Niveau der Kraftfähigkeiten des Oberkörpers (Liegestütze) und Rumpfs (Situps) sowie in der Beweglichkeit (Rumpfbeuge) dauerhaft über die Grundschulzeit zurückbleiben, haben ein höheres Risiko, muskuläre Dysbalancen $\mathrm{zu}$ entwickeln. Ružbarská (2016) konnte im Gegensatz zur vorliegenden Studie eindeutige Unterschiede für die Rumpfkraft bei den Sit-ups für Kinder mit normalem und verringertem Motorikindex nachweisen.

Fehlen die notwendigen Kraftfähigkeiten können sich Haltungsschwächen und im weiteren Verlauf Haltungsschäden bei den betroffenen Kindern manifestieren. So berichtet Raspe (2012), dass bereits 3\% der Drei- bis Sechsjährigen und 7\% der Sieben- bis Zehnjährigen an Rückenschmerzen leiden. Die Reduzierung muskulärer Dysbalancen ist deshalb ein zentrales Anliegen im Gesundheitssport (Rosenhagen, 2017).

Sowohl fein- als auch grobmotorisch auffällige Kinder hatten eine geringere aerobe Ausdauer anhand des SechsMinuten-Laufs als unauffällige Kinder. Diese Ausdauerleistung ist ein bedeutsamer Gesundheitsindikator und kennzeichnet das Niveau der kardiorespiratorischen Fitness (Gabrys, 2017). In der Folge dauerhaft niedriger Ausdauerleistungen über die Grundschulzeit, fehlen den Kindern die notwendigen Voraussetzungen für gesundheitswirksame moderate bis anstrengende Bewegungen. So erfüllen lediglich $46 \%$ der drei- bis sechsjährigen Kinder und $26 \%$ der sieben- bis zehnjährigen Kinder die gesundheitsbezogenen Bewegungsempfehlungen der WHO (Finger, Varnaccia, Borrmann et al., 2018). Abschließend unterstreichen die ANOVAR gestützten Unterschiede zu den motorischen Entwicklungsverläufen die prognostische Validität der Schuleingangsuntersuchung und der damit verbundenen Einschätzung des motorischen Entwicklungsstands.

\section{Schlussfolgerungen}

Vor dem Hintergrund, dass die motorischen Auffälligkeiten von Vorschulkindern über die Grundschulzeit als motorische Leistungsrückstände erhalten bleiben, stellt sich für die Praxis die Herausforderung, die bisherigen Ressourcen für förderbedürftige Kinder zu erhöhen. Hierzu sollten möglichst gleiche motorische Startbedingungen für die Kinder vor Schuleintritt das Ziel sein.

Eine weitere Perspektive ergibt sich für die gezielte Bewegungsförderung über die Grundschulzeit. Mit den Studienergebnissen liegen detaillierte Befunde zum Verlauf einzelner motorischer Fähigkeiten vor. Bewegungsfördernde Maßnahmen fallen aufgrund der raschen Lernfortschritte und Reifungsprozesse in dieser Entwick- 
lungsphase auf besonders fruchtbaren Boden (Albrecht et al., 2016). Bewegungsangebote für das mittlere Kindesalter sind vor allem reizvoll und motivierend durchzuführen, um unterschiedlichste Körpererfahrungen entstehen $\mathrm{zu}$ lassen. Im Vordergrund stehen die Vermittlung von Freude, die Stärkung des Selbstwertgefühls und die Erhöhung der Selbstständigkeit der Kinder (Winter \& Hartmann, 2015). Da sich bei den motorisch auffälligen Kindern die größten Defizite bei den koordinativen Fähigkeiten zeigten, bietet sich eine Schulung der Gleichgewichtsfähigkeit vorrangig in Spielform und bei sicherer Bewegungsbeherrschung eine Übungsgestaltung unter Zeitdruck an. Dies sollte an ein fortlaufendes Monitoring mit der Berücksichtigung motorischer Komponenten bei Leistungsüberprüfungen in der Schule geknüpft sein, um vor allem Kinder in der Schuleingangsphase bestmöglich unterstützen zu können (Bott, 2018). Zusätzlicher Forschungsbedarf ergibt sich für Analysen von Kindern, die gleichzeitig fein- und grobmotorische Störungen oder auch Auffälligkeiten in den emotionalen wie kognitiven Entwicklungsbereichen aufweisen. Aus dieser Erweiterung des Kenntnisstands zur motorischen Entwicklung können bewegungsbezogene Interventionen noch passgenauer entwickelt werden. Dies gilt auch für Fragestellungen, welche auf die Analyse motorischer Kompetenzen in Abhängigkeit der motorischen Voraussetzungen vor Schulbeginn ausgerichtet sind. Hierbei wäre zu klären, welche alltags- oder sportmotorischen Bewegungen mit einer reduzierten motorischen Leistungsfähigkeit assoziiert sind. Darüber hinaus sind Zusammenhangsanalysen mit der körperlich-sportlichen Aktivität der Kinder sowie konstitutionellen Merkmalen wie zum Beispiel dem Gewichtsstatus naheliegend, um weitere Einflussgrößen der motorischen Leistungsfähigkeit zu identifizieren.

\section{Elektronische Supplemente (ESM)}

Die elektronischen Supplemente sind mit der OnlineVersion dieses Artikels verfügbar unter https://doi.org/ 10.1026/0942-5403/a000351

ESM 1. Abbildung 1a-h. (Die Abbildungen zeigen den grafischen Verlauf der motorischen Fähigkeiten in $\mathrm{Ab}$ hängigkeit auffälliger motorischer Leistungen der Feinund Grobmotorik).

\section{Literatur}

Albrecht, C., Hanssen-Doose, A., Bös, K., Schlenker, L., Schmidt, S. C. E., Wagner, M. et al. (2016). Motorische Leistungsfähigkeit von
Kindern und Jugendlichen in Deutschland. Sportwissenschaft, 46, 294-304. https://doi.org/10.1007/s12662-016-0421-4

Baumann, N., Tresilian, J., Bartmann, P. \& Wolke, D. (2020). Early Motor Trajectories Predict Motor but not Cognitive Function in Preterm- and Term-Born Adults without Pre-existing Neurological Conditions. International journal of environmental research and public health, 17, 3258. https://doi.org/10.3390/ijerph17093258

Blank, R. \& Vinçon, S. (2020). Deutsch-österreichisch-schweizerische (DACH) Versorgungsleitlinie zu Definition, Diagnostik, Behandlung und psychosozialen Aspekten bei Umschriebenen Entwicklungsstörungen motorischer Funktionen (UEMF). Kurzfassung. Arbeitsgemeinschaft der Wissenschaftlichen Medizinischen Fachgesellschaften (AWMF online). Verfügbar unter https://www.awmf.org/ uploads/tx_szleitlinien/022-017k_S3_Umschriebene-Entwick lungsstoerungen-motorischer-Funktionen-UEMF_2020-08_01.pdf

Bös, K. (2016). Deutscher Motorik-Test 6-18. (DMT 6-18): Manual und internetbasierte Auswertungssoftware (Schriften der Deutschen Vereinigung für Sportwissenschaft, Bd. 186, 2. Aufl.). Hamburg: Feldhaus Edition Czwalina.

Bott, H. (2018). Motorisch ungeschickte (Schul)Kinder - Wie können wir sie erkennen? Potsdamer Zentrum für empirische Inklusionsforschung (ZEIF) - ZEIF Fachportal Wissenschaft - Praxis. https:// www.uni-potsdam.de/fileadmin/projects/inklusion/PDFs/ZEIFBlog/Bott_2018_Motorisch_ungeschickte_Kinder.pdf (9.7. 2021)

Cohen, J. (1988). Statistical Power Analysis for the Behavioral Sciences. Hillsdale, NJ: Erlbaum.

Dilling, H., Mombour, W., Schmidt, M. H. \& Schulte-Markwort, E. (Hrsg.). (2016). Internationale Klassifikationen psychischer Störungen. ICD-10 Kapitel V (F): Diagnostische Kriterien für Forschung und Praxis (6., überarb. Aufl. unter Berücksichtigung der Änderungen gemäß ICD-10-GM (German Modification) 2016). Bern: Hogrefe.

Döpfner, M., Dietmair, I., Mersmann, H., Simon, K. \& Trost-Brinkhues, G. (2004). S-ENS Screening des Entwicklungsstandes bei Einschulungsuntersuchungen. Instruktionen. Göttingen: Hogrefe.

Döpfner, M., Dietmair, l., Mersmann, H., Simon, K. \& Trost-Brinkhues, G. (2005). S-ENS Screening des Entwicklungsstandes bei Einschulungsuntersuchungen. Manual. Göttingen: Hogrefe.

Finger, J. D., Varnaccia, G., Borrmann, A., Lange, C. \& Mensink, G. B. M. (2018). Körperliche Aktivität von Kindern und Jugendlichen in Deutschland - Querschnittergebnisse aus KiGGS Welle 2 und Trends. Journal of Health Monitoring, 3, 24-31. https://doi.org/10. 17886/RKI-GBE-2018-006.2

Gabrys, L. (2017). Körperliche Fitness als gesundheitsrelevanter Prädiktor. In W. Banzer (Hrsg.), Körperliche Aktivität und Gesundheit. Präventive und therapeutische Ansätze der Bewegungs- und Sportmedizin (S. 67-75). Berlin: Springer.

Jaščenoka, J., Walter, F., Petermann, F., Korsch, F., Fiedler, S. \& Daseking, M. (2018). Zum Zusammenhang von motorischer und kognitiver Entwicklung im Vorschulalter. Kindheit und Entwicklung, 27, 142 - 152. https://doi.org/10.1026/0942-5403/a000254

Jekauc, D., Völkle, M., Lämmle, L. \& Woll, A. (2012). Fehlende Werte in sportwissenschaftlichen Untersuchungen. Sportwissenschaft, 42, 126-136. https://doi.org/10.1007/s12662-012-0249-5

Kastner, J. \& Petermann, F. (2010a). Entwicklungsbedingte Koordinationsstörungen. Zeitschrift für Sportpsychologie, 17, 36-49. https://doi.org/10.1026/1612-5010/a000007

Kastner, J. \& Petermann, F. (2010b). Entwicklungsbedingte Koordinationsstörungen und Lernverhalten. Monatsschrift Kinderheilkunde, 158, 455-462. https://doi.org/10.1007/s00112009-2124-5

Krombholz, H. (2015). Untersuchung der Entwicklung und Förderung von Kindern mit unterschiedlichem motorischem Leistungsstand im Vorschulalter. Zeitschrift für Sportpsychologie, 22, 63-76. https://doi.org/10.1026/1612-5010/a000142

Krug, S., Worth, A., Finger, J. D., Damerow, S. \& Manz, K. (2019). Motorische Leistungsfähigkeit 4- bis 10-jähriger Kinder in Deutsch- 
land: Ergebnisse aus KiGGS Welle 2 und Trends. Bundesgesundheitsblatt, Gesundheitsforschung, Gesundheitsschutz, 62, $1242-$ 1252. https://doi.org/10.1007/s00103-019-03016-7

Losse, A., Henderson, S. E., Elliman, D., Hall, D., Knight, E. \& Jongmans, M. (1991). Clumsiness in children-do they grow out of it? A 10-year follow-up study. Developmental medicine and child neurology, 33, 55-68. https://doi.org/10.1111/j.1469-8749. 1991.tb14785.x

Mair, P. \& Wilcox, R. (2020). Robust statistical methods in R using the WRS2 package. Behavior Research Methods, 52, 464-488. https://doi.org/10.3758/s13428-019-01246-w

Michel, E., Molitor, S., Fahmer, N. \& Schneider, W. (2019). Prognose motorischer Auffälligkeiten von Kita-Kindern für das Grundschulalter durch Einschätzungen von Eltern und pädagogischen Fachkräften. Frühe Bildung, 8, 73-80. https://doi.org/10.1026/21919186/a000421

Molitor, S., Michel, E. \& Schneider, W. (2015). Exekutive Funktionen bei Kindern mit motorischen Auffälligkeiten. Kindheit und Entwicklung, 24, 181-188. https://doi.org/10.1026/ 0942-5403/a000174

Oelze, J. (2014). Einflussfaktoren des Gewichtsstatus und der motorischen Leistungsfähigkeit im Einschulungsalter. Technische Universität Chemnitz. Chemnitz unter https://nbn-resolving.org/urn:nbn: de:bsz:ch1-qucosa-134808

Raspe, H. (2012). Rückenschmerzen (Gesundheitsberichterstattung des Bundes, Bd. 53). Berlin: Robert-Koch-Institut.

Rivilis, I., Hay, J., Cairney, J., Klentrou, P., Liu, J. \& Faught, B. E. (2011). Physical activity and fitness in children with developmental coordination disorder: a systematic review. Research in developmental disabilities, 32, 894-910. https://doi.org/10.1016/j.ridd.2011.01. 017

Rosenhagen, A. (2017). Bewegung und Gesundheit im Kindesalter. In W. Banzer (Hrsg.), Körperliche Aktivität und Gesundheit. Präventive und therapeutische Ansätze der Bewegungs- und Sportmedizin (S. 124-137). Berlin: Springer.

Roth, A., Schmidt, S. C. E., Seidel, I., Woll, A. \& Bös, K. (2018). Tracking of Physical Fitness of Primary School Children in Trier. A 4-Year Longitudinal Study. BioMed research international, 2018, 1-10. https://doi.org/10.1155/2018/7231818

Ružbarská, I. (2016). Physical fitness of primary school children in the reflection of different levels of gross motor coordination. Acta Gymnica, 46, 184-192. https://doi.org/10.5507/ag.2016.018

Scheuer, C., Herrmann, C. \& Bund, A. (2019). Motor tests for primary school aged children: A systematic review. Journal of sports sciences, 37, 1097-1112. https://doi.org/10.1080/02640414.2018. 1544535

Schnabel, G. (2015). Bewegungskoordination als Regulation der Bewegungstätigkeit. In K. Meinel \& G. Schnabel (Hrsg.), Bewegungslehre - Sportmotorik. Abriss einer Theorie der sportlichen Motorik unter pädagogischem Aspekt (12., erg. Aufl., S. 28-71). Aachen: Meyer \& Meyer Verlag.
Speer, A., Wagner, P., Streicher, H., Ziegeldorf, A. \& Wulff, H. (2020). KOMPASS-2-Studie - Motorische Leistungsfähigkeit von Grundschülern und Impulse für Interventio-nen. In P. Neumann \& E. Balz (Hrsg.), Grundschulsport. Empirische Einblicke und pädagogische Empfehlungen (Edition Schulsport, S. 66-81). Aachen: Meyer \& Meyer.

Streicher, H., Wulff, H., Hartmann, C., Witt, M. \& Wagner, P. (2017). Die Leipziger KOMPASS(2)-Studie: Einführung und Studiendesign. In M. Busse (Hrsg.), Leipziger Sportwissenschaftliche Beiträge. Schwerpunktthemen: Kinder und Bewegung, Motorische Tests (Bd. 1, S. 158-176). Berlin: Lehmanns.

Utesch, T., Strauß, B., Tietjens, M., Büsch, D., Ghanbari, M.-C. \& Seidel, I. (2015). Die Überprüfung der Konstruktvalidität des Deutschen Motorik-Tests 6-18 für 9- bis 10-Jährige. Zeitschrift für Sportpsychologie, 22, 77-90. https://doi.org/10.1026/1612-5010/ a000143

Utesch, T., Zinner, J. \& Büsch, D. (2018). Stabilität der physischen Fitness im Kindesalter. German Journal of Exercise and Sport Research, 48, 404-414. https://doi.org/10.1007/s12662-018-0500-9

Werpup-Stüwe, L. \& Petermann, F. (2015). Visuelle Wahrnehmungsleistungen bei motorisch auffälligen Kindern - eine Pilotstudie. Praxis der Kinderpsychologie und Kinderpsychiatrie, 64, 601-616. https://doi.org/10.13109/prkk.2015.64.8.601

Winter, R. \& Hartmann, C. (2015). Die motorische Entwicklung (Ontogenese) des Menschen (Überblick). In K. Meinel \& G. Schnabel (Hrsg.), Bewegungslehre - Sportmotorik. Abriss einer Theorie der sportlichen Motorik unter pädagogischem Aspekt (12., erg. Aufl., S. 243 - 373). Aachen: Meyer \& Meyer.

Woll, A., Worth, A., Bös, K. \& MoMo-Team. (2019). MoMo NEUE DATEN FÜR TATEN. Ergebnisse aus der Motorik-Modul-Längsschnittstudie (MoMo). Karlsruhe: Karlsruher Institut für Technologie (KIT).

\section{Historie}

Onlineveröffentlichung: 03.09. 2021

\section{Interessenskonflikt}

Die Autoren geben an, dass kein finanzieller oder firmenbezogener Interessenkonflikt besteht. Die Studie wurde durch die Ethikkommissionen der Medizinischen Fakultät der Universität Leipzig geprüft und als unbedenklich genehmigt.

\section{Förderung}

Open Access-Veröffentlichung ermöglicht durch die Universität Leipzig.

\section{Andreas Speer}

Institut für Gesundheitssport und Public Health Universität Leipzig, Sportwissenschaftliche Fakultät Jahnallee 59

04109 Leipzig

andreas.speer@uni-leipzig.de 